\title{
A Murgnahan-Nakayama rule for Schubert polynomials
}

\author{
Andrew Morrison $]^{k}$ \\ Departement Mathematik, ETHZ, Rämistrasse 101, Zurich 8092, Switzerland
}

\begin{abstract}
We expose a rule for multiplying a general Schubert polynomial with a power sum polynomial in $k$ variables. A signed sum over cyclic permutations replaces the signed sum over rim hooks in the classical MurgnahanNakayama rule. In the intersection theory of flag manifolds this computes all intersections of Schubert cycles with tautological classes coming from the Chern character. We also discuss extensions of this rule to small quantum cohomology.

Résumé. Nous ècrivons une formule pour multiplier les polynômes de Schubert avec les sommes de Newton. Une somme signée de permutations cycliques remplace la somme signée de rubans dans la formule classique de MurgnahanNakayama. Nous obtenons donc des relations dans l'anneau de Chow de la variété de drapeaux. Nous discutons également des extensions de cette formule en cohomologie quantique.
\end{abstract}

Keywords: Murnaghan-Nakayama formula, Schubert polynomials, quantum cohomology.

\section{Introduction}

To each partition $\lambda$ we may associate a Schur function $s_{\lambda}$, which is a formal generating series for all semi-standard Young tableaux of shape $\lambda$. These form an integer basis for the algebra of symmetric functions. Alternatively, the symmetric functions (over $\mathbb{Q}$ ) are freely generated as an algebra by the power sum symmetric functions $p_{r}$.

The classical Murnaghan-Nakayama rule expresses the product of a Schur function and a power sum function in the Schur basis,

$$
p_{r} \cdot s_{\lambda}=\sum_{\mu}(-1)^{h t(\mu / \lambda)} s_{\mu},
$$

the sum is over all partitions $\mu$ such that $\mu / \lambda$ is a rim hook of length $r$ and ht $(\mu / \lambda)$ is its height [21].

When we rewrite the Schur functions as linear combinations of products of power sum functions the change of basis matrix is given by the characters of the symmetric group. In this way the above algebrocombinatorial relation is equivalently expressed in representation theory as a character formula [10].

*Email: andrewmo@math. ethz.ch. Supported by the Swiss National Science Foundation through grant SNF-200021143274 and the MSRI.

1365-8050 (c) 2014 Discrete Mathematics and Theoretical Computer Science (DMTCS), Nancy, France 
The Murnaghan-Nakayama rule also has geometric content. The ring of symmetric functions provides an algebraic model for the Chow/Cohomology ring of the Grassmannian. The associated Schur polynomials represent geometric degeneracy loci while the power sum polynomials give Chern characters of the tautological bundle. The intersection of these cycles then has significance for enumerative geometry [11].

The goal of the paper is to write down the analogous formula for Schubert polynomials:

Theorem 1. For any permutation $\omega$ we have

$$
p_{r}\left(x_{1}, \ldots, x_{k}\right) \cdot \mathfrak{S}_{\omega}=\sum(-1)^{\epsilon_{k}(\sigma)} \mathfrak{S}_{\omega \cdot \sigma},
$$

the sum over all cycles $\sigma$ such that

$$
\omega<_{k} \omega \cdot \sigma \text { and } l(\omega \cdot \sigma)=l(\omega)+r
$$

where $<_{k}$ is the $k$-Bruhat order, $l(\omega)$ is the length of $\omega$, and

$$
\epsilon_{k}(\sigma):=\#\{i \leq k: \sigma(i) \neq i\}+1 .
$$

In the final section of this abstract we discuss extensions of this rule to (small) quantum cohomology.

Several generalizations of the Murnaghan-Nakayama rule have appeared in the literature. Fomin and Green gave a version for certain non-commutative symmetric functions, which led to combinatorial formulas for characters of representations associated to stable Schubert and stable Grothendieck polynomials [12]. McNamara gave a skew version of the Murnaghan-Nakayama rule [23], which Konvalinka generalized to a skew rule for multiplication by a 'quantum' (perturbed with parameter $q$ ) power sum function [16]. Bandlow, et al. gave a version of it in the cohomology of an affine Grassmannian [1]. That is, a formula for the product of a power sum symmetric function by a $k$-Schur function expanded in the basis of $k$-Schur polynomials. In another recent paper Ross gave a new proof of the MurnaghanNakayama rule which generalized to loop Schur functions [27] thus proving a fundamental step in the orbifold GW/DT correspondence [28].

\section{The Murgnahan-Nakayama rule for Schubert polynomials.}

The Schubert polynomials of Lascoux and Schützenberger [19] provide a linear basis for the space of all polynomials in a countable number of variables. This Schubert basis contains all Schur symmetric polynomials. While the well-known Littlewood Richardson rule describes products of Schur polynomials, it is a open problem to give a similar combinatorial expression for the multiplication of Schubert polynomials [22].

We begin by recalling the definition of Schubert polynomials. Then we explain how the Schur polynomials embed in the ring of Schubert polynomials and give the known rules for multiplying a Schur polynomial and a Schubert polynomial. We then give a short proof of the Murgnahan-Nakayama rule for Schubert polynomials and end by explaining a geometric application to the intersection theory of flag manifolds, which was the motivation for this work.

\subsection{Preliminaries.}

Bruhat order. Write $s_{a b}$ for the transposition in the symmetric group $S_{n}$ interchanging integers $a$ and $b$ and $s_{i}$ for $s_{i i+1}$. Every permutation admits a factorization as $\omega=s_{a_{1}} \cdots s_{a_{r}}$. When this is of minimal 
length we call $\left(a_{1}, \ldots, a_{r}\right)$ a reduced word for $\omega$. The length of this word is the number of inversions in $w$, i.e. pairs $i<j$ such that $\omega(i)>\omega(j)$, we denote this number by $l(\omega)$. The Bruhat order on permutations is generated by the following covering relation

$$
\omega \lessdot \omega^{\prime} \text { if } \omega^{\prime}=\omega \cdot s_{a b} \text { and } l\left(\omega^{\prime}\right)=l(\omega)+1 .
$$

The $k$-Bruhat order $<_{k}$ is the suborder whose covers are as above where $a \leq k<b$.

Schubert polynomials. The group $S_{n}$ acts on $R_{n}=\mathbb{Z}\left[x_{1}, \ldots, x_{n}\right]$ by permuting the variables. Define the divided difference operators $\partial_{i}$ for $i=1, \ldots, n-1$ by

$$
\partial_{i}=\frac{1-s_{i}}{x_{i}-x_{i+1}}
$$

where the transposition $s_{i}$ interchanges $x_{i}$ and $x_{i+1}$ leaving the other generators invariant. We have $\partial_{i} \circ \partial_{i}=0$ and these operators satisfy the braid relations so that if $\omega$ has reduced word $\left(a_{1}, \ldots, a_{r}\right)$ then $\partial_{\omega}:=\partial_{a_{1}} \circ \cdots \circ \partial_{a_{r}}$ does not depend upon the choice of reduced word. Lascoux and Schützenberger defined the Schubert polynomial $\mathfrak{S}_{\omega}$ as

$$
\mathfrak{S}_{\omega}=\partial_{\omega^{-1} \omega_{0}}\left(x_{1}^{n-1} x_{2}^{n-2} \cdots x_{n-1}\right),
$$

were $w_{0}$ is the involution sending $i$ to $n-i+1$. If we let $I_{n}$ be the ideal generated by the non-constant symmetric functions then the above Schubert polynomials form a basis for $H_{n}=R_{n} / I_{n}$.

Schur polynomials from Grassmannian permutations. A permutation $\omega \in S_{n}$ is called Grassmannian of descent $k$ if $\omega(i)<\omega(i+1)$ for $i \neq k$. Such a permutation $\omega$ determines a partition

$$
\lambda=\lambda(\omega)=(\omega(k)-k, \omega(k-1)-k+1, \ldots, \omega(1)-1) .
$$

Schubert polynomials corresponding to Grassmannian permutations give the Schur polynomials. Specifically, if $\omega$ is a Grassmannian permutation with descent $k$ and partition $\lambda$, then

$$
\mathfrak{S}_{\omega}=s_{\lambda}\left(x_{1}, \ldots, x_{k}\right) \text {. }
$$

Pieri's rule for Schubert polynomials. The classical Pieri's rule gives a rule for multiplying a complete symmetric function $h_{r}=s_{(r)}$ with a Schur function $s_{\lambda}$,

$$
h_{r} \cdot s_{\lambda}=\sum_{\mu} s_{\mu},
$$

where the sum is over $\mu$ such that no two boxes in the diagram of $\mu / \lambda$ appear in the same column. Using the involution $w$ on the space of symmetric functions defined by $w\left(h_{r}\right)=e_{r}$, we have $w\left(s_{\lambda}\right)=s_{\lambda^{t}}$ and an equivalent statement can be deduced for the elementary symmetric functions.

The first such rule for Schubert polynomials appeared in a paper of Monk [25]. Monk showed that for the transposition $s_{k}=(k k+1)$ one gets

$$
\mathfrak{S}_{s_{k}} \cdot \mathfrak{S}_{\omega}=\sum_{\substack{\omega<k \omega^{\prime} \\ l\left(\omega^{\prime}\right)=l(\omega)+1}} \mathfrak{S}_{\omega^{\prime}} .
$$


From the definition it is an easy exercise to show that

$$
\mathfrak{S}_{s_{k}}=e_{1}\left(x_{1}, \ldots, x_{k}\right)=x_{1}+\cdots+x_{k} .
$$

Monk's rule determines all the structure constants for the multiplication of Schubert polynomials. The product of the $r$ th elementary symmetric polynomial in $k$ variables with a Schubert polynomial was formulated by Lascoux and Schützenberger [19] in analogy with the classical Pieri rule above. This was proven geometrically by Sottile [29], where he also gave a generalization to hook Schur functions.

Theorem 2. ([29], Theorem 8) Let $q \leq k$ and $k+p \leq n$ be integers and set $m=p+q-1$. For $\omega \in S_{n}$,

$$
s_{\left(p, 1^{q-1}\right)}\left(x_{1}, \ldots, x_{k}\right) \cdot \mathfrak{S}_{\omega}=\sum \mathfrak{S}_{\operatorname{end}(\gamma)}
$$

the sum over all paths $\gamma: \omega<_{k} \omega^{(1)}<_{k} \cdots<_{k} \omega^{(m)}$ in the $k$-Bruhat order such that on writing $\omega^{(i)}=\omega^{(i-1)} \cdot\left(a_{i} b_{i}\right)$ with $a_{i}<b_{i}$ we have

$$
\omega^{(1)}\left(a_{1}\right)>\cdots>\omega^{(q)}\left(a_{q}\right) \text { and } \omega^{(q)}\left(a_{q}\right)<\cdots<\omega^{(m)}\left(a_{m}\right) .
$$

Setting $p$ or $q$ equal to 1 in the above gives the Schubert polynomial analogue of the classical Pieri rule.

\subsection{Proof of the Murgnahan-Nakayama rule for Schubert polynomials.}

We first apply Monk's formula to deduce that only terms coming from cycles occur. Then we use the expression for the power sum polynomial as an alternating sum of hook Schur polynomials. Using Sottile's Pieri rule shows that the contribution from each hook Schur function is expressed as a count of paths in the $k$-Bruhat order, then we apply a result of Begeron and Sottile [2] for paths in the $k$-Bruhat order to see that each cycle contributes only once.

Step one. First we use Monk's formula to see that the answer can be written as a sum of permutations that differ by a cycle. Monk's formula can equivalently be stated as [22]

$$
x_{k} \cdot \mathfrak{S}_{\omega}=\sum_{l\left(\omega \cdot s_{k b}\right)=l(\omega)+1} \mathfrak{S}_{\omega \cdot s_{k b}}-\sum_{l\left(\omega \cdot s_{a k}\right)=l(\omega)+1} \mathfrak{S}_{\omega \cdot s_{a k}} .
$$

Applying this $r$ times gives a sum over cycles

$$
x_{k}^{r} \cdot \mathfrak{S}_{\omega}=\sum_{\sigma=\left(k, c_{1}, \ldots, c_{r}\right)} a_{\sigma} \mathfrak{S}_{\omega \cdot \sigma}
$$

where $a_{\sigma} \in \mathbb{Z}$, so that ultimately $p_{r}\left(x_{1}, \ldots, x_{k}\right) \cdot \mathfrak{S}_{\omega}$ is a sum over cycles.

Step two. We next rewrite the power sum symmetric function as an alternating sum of hook Schur functions

$$
p_{r}=\sum_{p+q-1=n}(-1)^{p} s_{\left(p, 1^{q-1}\right)} .
$$

This is a simple case of the Murgnahan-Nakayama rule for symmetric functions. It now suffices to prove the following claim

$$
s_{\left(p, 1^{q-1}\right)} \cdot \mathfrak{S}_{\omega}=\sum_{\substack{\sigma-c y c l e \\ \omega<k \\ l(\omega \cdot \sigma)=l(\omega)+r \\ \\ \epsilon_{k}(\sigma)=p}} \mathfrak{S}_{\omega \cdot \sigma}+\sum \ldots
$$


where here the second sum contains contributions from not arising from cycles, that is, terms $\mathfrak{S}_{\omega^{\prime}}$ such that $\omega^{-1} \cdot \omega^{\prime}$ is not a cycle.

Step three. Finally we compute the contribution from each hook Schur function. We begin by showing that the cycles $\sigma$ occurring in the product of $s_{\left(p, 1^{q-1}\right)}$ and $\mathfrak{S}_{\omega}$ all have $\epsilon_{k}(\sigma)=p$. This follows by induction. In the special case of complete symmetric and elementary symmetric polynomials we have the Pieri formulas

$$
\begin{aligned}
s_{\left(1^{q}\right)}\left(x_{1}, \ldots, x_{k}\right) \cdot \mathfrak{S}_{\omega} & =\sum_{\gamma: a_{i} \neq a_{j} \forall i \neq j} \mathfrak{S}_{\operatorname{end}(\gamma)} \\
s_{(p)}\left(x_{1}, \ldots, x_{k}\right) \cdot \mathfrak{S}_{\omega} & =\sum_{\gamma: b_{i} \neq b_{j} \forall i \neq j} \mathfrak{S}_{\operatorname{end}(\gamma)}
\end{aligned}
$$

Considering these together we see that in the product

$$
s_{(p)}\left(x_{1}, \ldots, x_{k}\right) \cdot s\left(1^{q}\right)\left(x_{1}, \ldots, x_{k}\right) \cdot=\mathfrak{S}_{\omega}=\sum_{\omega^{\prime}} a_{\omega^{\prime}} \mathfrak{S}_{\omega^{\prime}}
$$

two types of $p+q-1$-cycle $\sigma$ with $\omega^{\prime}=\omega \cdot \sigma$ can occur. Firstly those with $p+1$ district entries $a_{i}$ less than of equal to $k$, and secondly those with $p$ distinct entries $a_{i}$ less than or equal to $k$. Invoking the identity

$$
s_{(p)}(x) \cdot s_{\left(1^{q}\right)}(x)=s_{\left(p+1,1^{q}\right)}(x)+s_{\left(p, 1^{q-1}\right)}(x)
$$

one finds, by induction on $p$, that these correspond firstly to the contribution of cycle terms from $s_{\left(p+1,1^{q}\right)}(x)$ and secondly to the contribution of cycle terms from $s_{\left(p, 1^{q-1}\right)}(x)$.

Thus the contribution to $s_{\left(p, 1^{q-1}\right)} \cdot \mathfrak{S}_{\omega}$ coming from cycles is

$$
\sum_{\substack{\sigma-\text { cycle } \\ \omega<k \omega \cdot \sigma \\ l(\omega \cdot \sigma)=l(\omega)+r \\ \epsilon_{k}(\sigma)=p}} n(\sigma) \cdot \mathfrak{S}_{\omega \cdot \sigma}
$$

where

$$
n(\sigma)=\#\left\{\gamma: \begin{array}{l}
\text { paths } \gamma: \omega<_{k} \omega^{(1)}, k \cdots<_{k} w^{(r)}=\omega \cdot \sigma \\
\omega^{(1)}\left(a_{1}\right)>\cdots>\omega^{(q)}\left(a_{q}\right)<\cdots \omega^{(r)}\left(a_{r}\right)
\end{array}\right\} .
$$

The result of [2, Lemma 6.8] implies that there is a unique path from $w$ to $w \sigma$ in the $k$-Bruhat order whose sequence of labels $\left\{\omega^{(i)}\left(a_{i}\right)\right\}$ was peakless. This implies that $n(\sigma)=1$. Note: to use [2, Lemma 6.8] here, we need to note that the cycle it uses is $\omega \sigma \omega^{-1}$ and that the path will end in an increasing sequence of $\epsilon_{k}(\sigma)$ labels.

\subsection{Application to flag manifolds.}

The manifold of complete flags $F l(n)$ has a modular interpretation with a universal family of flags

$$
F l(n): \mathcal{F}_{0} \subset \mathcal{F}_{1} \subset \cdots \subset \mathcal{F}_{n}=\mathbb{C}^{\times n} \times F l(n)
$$

where $\mathcal{F}_{i}$ is the rank $i$ tautological bundle given by the $i$ th flag. We denote by $x_{i}=c_{1}\left(\mathcal{F}_{i} / \mathcal{F}_{i+1}\right), i=$ $1, \ldots, n$. These algebraic classes generate the cohomology of $F l(n)$ and the only relations between them are the non-constant symmetric functions in $x_{1}, \ldots, x_{n}$. 
Geometrically $F l(n)$ has a cell decomposition. We fix a specific flag $F^{\bullet}$, so that $F_{i}=\left\langle e_{1}, \ldots, e_{i}\right\rangle$ and $\hat{F}_{n-i}=\left\langle e_{i+1}, \ldots, e_{n}\right\rangle$ then

$$
\left.X_{\omega}^{0}:=\left\{E^{\bullet} \in F l(n): \operatorname{dim}\left(E_{p} \cap \hat{F}_{q}=\#\{i \leq p: \omega(i) \geq n+1-q\} \forall p, q\right\}\right)\right\}
$$

each such cell has co-dimension $\ell(\omega)$ and we get a geometric basis for the cohomology given by

$$
H^{*}(F l(n))=\oplus_{\omega \in S_{n}} \mathbb{Z}\left[\bar{X}_{\omega}^{0}\right] .
$$

It was shown by Bernstein-Gel'fand-Gel'fand [3] and Demazure [8] that by identifying $x_{i}$ with the first Chern class as above these geometric Schubert cells are represented in cohomology by the corresponding Schubert polynomials so that we have a natural isomorphism of rings $H^{*}(F l(n))=H_{n}$ above. This gives a geometric interpretation to all terms in our Murgnahan-Nakayama rule. That is,

$$
\mathfrak{S}_{\omega}=\left[\bar{X}_{\omega}^{0}\right] \quad \text { and } \quad p_{r}\left(x_{1}, \ldots, x_{k}\right)=r ! \cdot c h_{r}\left(\mathcal{F}_{k}\right)
$$

and we can interpret the formula as a geometric representation of the intersection of a Schubert cell with the $r$ th Chern character of one of the tautological bundles.

Remark. The above formulas determine the intersection number of Schubert cells and Chern characters in any partial flag manifold. This is because the cohomology of the partial flag manifold is a subring of the cohomology of the complete flag manifold. Also in the above we worked with the singular cohomology of the complex flag variety but everything is valid for the Chow rings with $\mathbb{Q}$ coefficients of flag varieties defined over any base field.

Remark. As another possible application we remark that the number of terms occurring in the MurnaghanNakayama rule is vastly smaller than those occurring in the Pieri rule. Potentially, this could be exploited for efficient computations as in classical case where the rule provides an effective method for inductively computing the characters of the symmetric group.

\section{The Murnagahan-Nakayama rule in quantum cohomology.}

The (small) quantum cohomology ring of a flag manifold gives a deformation of the classical cohomology so that additively we have,

$$
q H^{*}\left(F l\left(d_{1}<\cdots<d_{k}<n\right)=H^{*}\left(F l\left(d_{1}<\cdots<d_{k}<n\right)\right) \otimes \mathbb{Q}\left[q_{1}, \ldots, q_{k}\right] .\right.
$$

However the multiplicative structure is non-trivial. For example, the construction is not functorial so that we cannot embed the quantum cohomology of a partial flag manifold inside the quantum cohomology of the complete flag manifold.

In the case of the Grassmannian there is a rim hook algorithm which essentially enables us to express the quantum product in terms of the classical one. Using this we write down a simple Murnagahan-Nakayama rule in the quantum cohomology of the Grassmannian.

\subsection{Quantum cohomology.}

Definition. Let $X$ be a complex projective manifold. Suppose that $\left\{T^{i}\right\}$ and $\left\{T_{i}\right\}$ are dual basis for the classical cohomology $H^{*}(X)$. The quantum product of two cohomology classes is defined in terms of 
three point Gromov-Witten invariants

$$
a *_{q} b=\sum_{\beta \in H_{2}}\left\langle a, b, T_{i}\right\rangle_{0,3, \beta} q^{\beta} T^{i}
$$

where the sum is over all $i$, all effective classes $\beta$ and $q$ is as a formal parameter of the generating series. The three point function $\langle\bullet, \bullet, \bullet\rangle_{0,3, \beta}$ is defined by an integral over the moduli space of stable maps $\overline{\mathcal{M}}_{0,3}(X, \beta)$ and gives a virtual count of the number of rational curves in the class $\beta$ intersecting $a, b$ and $T_{i}$ [14]. In particular the constant term of the series is the classical product so setting $q=0$ we recover the ordinary cohomology.

Grassmannian. Grassmannians were the first collection of manifolds to have their quantum cohomology computed [31,4]. The ring structure is given by

$$
q H^{*}(G r(k, n))=\mathbb{Q}\left[e_{1}, \ldots, e_{k}\right][q] /\left(h_{n-k+1}, \ldots, h_{n-1}, h_{n}+(-1)^{k} q\right)
$$

Here $e_{i}$ is the $i$ th elementary symmetric polynomial in $k$ variables and $h_{i}$ is the $i$ th complete symmetric polynomial in $k$ variables. From this we can immediately see that the $q \rightarrow 0$ limit recovers the classical cohomology. It was shown in [4] that the geometric Schubert cell in the Grassmannian corresponding to the partition $\lambda$ is represented in this ring by the Schur polynomial $s_{\lambda}$ in $k$ variables. In particular the $e_{i}$ and $h_{i}$ correspond to the $i$ th Chern classes of the tautological and quotient bundles respectively.

We can express this quantum cohomology ring as a quotient in another way. Let $\Lambda_{k}=\mathbb{Q}\left[e_{1}, \ldots, e_{k}\right]$ be the ring of symmetric polynomials in $k$ variables. Sending $e_{i}$ to the $i$ th Chern character of the tautological bundle and $s_{\left(n-k+1,1^{k-1}\right)}$ to the quantum parameter $q$ gives an isomorphism

$$
\psi: \Lambda_{k} /\left(h_{n-k+1}, \ldots, h_{n-1}\right) \stackrel{\sim}{\longrightarrow} q H^{*}(G r(k, n)) .
$$

To see that this onto map is an isomorphism we consider the identity

$$
h_{a}-e_{1} h_{a-1}+e_{2} h_{a-2}-\cdots+(-1)^{a} e_{a}=0
$$

which specializes when $a=n$ to

$$
h_{n}+(-1)^{k} s_{\left(n-k+1,1^{k-1}\right)}=0
$$

since $e_{k} h_{n-k}=s_{\left(n-k+1,1^{k-1}\right)}$ in $\Lambda_{k, n}:=\Lambda_{k} /\left(h_{n-k+1}, \ldots, h_{n-1}\right)$.

\subsection{The Murnagahan-Nakayama rule in $q H^{*}(G r(k, n))$}

One obtains a Murnagahan-Nakayama rule for the quantum cohomology of the Grassmannian by analysing the terms in the classical Murnagahan-Nakayama rule under the rim hook algorithm.

Proof via rim hook algorithm. The strategy of proof is to take $s_{\lambda} \in \Lambda_{k, n}$ lift this to $s_{\lambda} \in \Lambda_{k}$ then compute $p_{r} \cdot s_{\lambda}$ in $\Lambda_{k}$ where we have the classical Murnaghan-Nakayama rule. We push forward the resulting alternating sum to $\Lambda_{k, n}$, and then consider the class in $q H^{*}(G r(k, n))$. As such it suffices to compute the image of an arbitrary Schur polynomial under the map $\Lambda_{k} \rightarrow \Lambda_{k, n} \rightarrow q H^{*}(G r(k, n))$.

In their study of representations of Hecke algebras at $n$th roots of unity, Goodman and Wenzl [15] defined a (seemingly) different quotient of $\Lambda_{k}$,

$$
\Lambda_{k} /\left(s_{\lambda}: \lambda_{1}-\lambda_{k}=n-k+1\right),
$$


after rewriting the partitions in shifted Frobenius coordinates the relation $\lambda_{1}-\lambda_{k}=n-k+1$ they get an action of the affine reflection group on $\mathbb{R}^{k}$ generated by the symmetric group $S_{k}$ and one additional reflection. Certain orbits of the reflection group action give a basis for the quotient ring, from this they found a formula for the corresponding Littlewood-Richardson coefficients in their quotient ring which was equal to a formula obtained by Kac [17] and Walton [30] for fusion coefficients in a Wess-Zumino-Witten conformal field theory. Later, Bertram, Ciocan-Fontanine and Fulton [5] obtained the same formula for the Littlewood-Richardson coefficients in the quantum cohomology of the Grassmannian, which shows that this quotient is equal to the quantum cohomology ring $q H^{*}(\operatorname{Gr}(k, n))$ and the ideals in both quotients coincide.

From this re-expression of the quantum cohomology ring the image of a Schur polynomial $s_{\lambda} \in \Lambda_{k}$ in $q H^{*}(G r(k, n))$ can now be written in a simple combinatorial way. Given any partition $\lambda$ with at most $k$ parts, we define its $n$-core $\hat{\lambda}$ by repeatedly removing rim hooks of length $n$ until it is not possible to remove any more, what results is a well defined partition independent of the choices of rim hooks [21]. The image of a Schur polynomial $s_{\lambda} \in \Lambda_{k}$ in $q H^{*}(G r(k, n))$ is given in [5] by

$$
s_{\lambda} \longmapsto\left\{\begin{array}{cl}
\epsilon(\lambda / \hat{\lambda}) q^{(|\lambda|-|\hat{\lambda}|) / n} s_{\hat{\lambda}} & \text { if } \hat{\lambda}_{1} \leq n-k \\
0 & \text { if } \hat{\lambda}_{1}>n-k
\end{array}\right.
$$

where $\epsilon(\lambda / \hat{\lambda})$ is a sign with parity $\sum k-h t\left(r_{i}\right)$ summed over all rim hooks $r_{i}$ removed. The MurnaghanNakayama rule for $q H^{*}(G r(k, n))$ is an immediate consequence of this algorithm:

Theorem 3. [[5]] Let $\lambda \subset\left(k^{n-k}\right)$ be a partition and $r \leq n$ be a positive integer. Then

$$
p_{r} *_{q} s_{\lambda}=\sum_{\mu}(-1)^{h t(\mu / \lambda)} s_{\mu}-(-1)^{k} q \sum_{\nu}(-1)^{h t(\lambda / \mu)} s_{\nu}
$$

where the first sum is over all $\mu \subset\left(k^{n-k}\right)$ with $\mu / \lambda$ a rim hook of size $r$ and the second sum is over all $\nu \subset\left(k^{n-k}\right)$ with $\lambda / \nu$ a rim hook of size $n-r$.

Proof. We first of all lift the calculation to $\Lambda_{k}$ where the product has the usual classical expansion,

$$
p_{r} s_{\lambda}=\sum_{\mu}(-1)^{h t(\mu / \lambda)} s_{\mu}
$$

given by summing over all the relevant Schur polynomials in $\Lambda_{k}$. To discover the image of these Schur polynomials in the quotient we apply the rim hook algorithm. There are two cases. When $\mu \subset\left(k^{n-k}\right)$, it is already an $n$-core, so in the quotient these terms give the classical contribution occurring in the first part of the sum. When $\mu$ is not contained in $\left(k^{n-k}\right)$, the rim hook algorithm removes all boxes outside of $\left(k^{n-k}\right)$. The only way to remove a length $n$-rim hook from $\mu$ is to start at the corner of the first column. Any other rim hook would have smaller length by construction of $\mu$. If this $n$-rim hook cannot be removed then $\mu$ is already an $n$-core and has image zero in the quotient. Otherwise the effect of removing the $n$-rim hook from the partition $\mu$ can been seen as removing an $n-k$-rim hook from the original partition $\lambda$, in the quotient this term comes with a linear $q$ factor and a sign. Moreover any such term can be achieved in this way giving the second sum in the formula. 


\subsection{The Murnagahan-Nakayama rule in $q H^{*}(F l(n))$.}

Postnikov [26] gave a quantum version of Pieri's formula for $F l(n)$ and Ciocan-Fontanine gave quantum Pieri rules for all partial flag manifolds. Quantum Chevalley formulas for general $G / P$ were given by Woodward and Fulton in [9] proving a generalization of Peterson's formula.

The quantum cohomology ring of the complete flag manifold was computed by Kim [18] and CiocanFontanine [7]

$$
q H^{*}(F l(n))=\mathbb{Q}\left[x_{1}, \ldots, x_{n}, q_{1}, \ldots, q_{n-1}\right] /\left(E_{1}, \ldots, E_{n}\right) .
$$

here $E_{i}\left(x_{1}, \ldots, x_{n}\right)$ is the $i$ th coefficient in the characteristic polynomial of

$$
\left(\begin{array}{ccccc}
x_{1} & q_{1} & 0 & \cdots & 0 \\
-1 & x_{2} & q_{2} & \cdots & 0 \\
0 & -1 & x_{3} & \cdots & 0 \\
\vdots & \vdots & \vdots & \ddots & \vdots \\
0 & 0 & 0 & \cdots & x_{n}
\end{array}\right) .
$$

The goal of this section is to write down a Murgnahan-Nakayama rule in the quantum cohomology of the complete flag manifold.

Quantum polynomials. In the quantum cohomology of the complete flag manifold the Schubert cells $\bar{X}_{\omega}^{0}$ are represented by quantum Schubert polynomials $\mathfrak{S}_{\omega}^{q}(x)$ in $q H^{*}(F l(n))$ [13]. For $J=\left(j_{1}, \ldots, j_{n-1}\right)$ and writing $e_{J}$ and $E_{J}$ for the polynomials

$$
e_{J}=\prod_{i=1}^{n-1} e_{j_{i}}\left(x_{1}, \ldots, x_{i}\right) \quad \text { and } \quad E_{J}=\prod_{i=1}^{n-1} E_{j_{i}}\left(x_{1}, \ldots, x_{i}\right)
$$

then each (classical) Schubert polynomial has a unique expression as $\mathfrak{S}_{\omega}^{q}(x)=\sum a_{J} e_{J}(x)$ with $a_{J} \in \mathbb{Z}$. The quantum Schubert polynomials are then defined by

$$
\mathfrak{S}_{\omega}^{q}(x)=\sum a_{J} E_{J}(x) .
$$

For a Grassmannian permutation corresponding to a partition $\lambda$ of descent $k$ alternatively denote this quantum deformation of the Schur polynomial as $s_{\lambda}^{q}\left(x_{1}, \ldots, x_{k}\right)$. We define our quantum power sum polynomial in analogy with the classical case as the alternating sum of quantum hook Schur polynomials,

$$
p_{r}^{q}\left(x_{1}, \ldots, x_{k}\right)=s_{(r)}^{q}\left(x_{1}, \ldots, x_{k}\right)-s_{(r-1,1)}^{q}\left(x_{1}, \ldots, x_{k}\right)+\cdots+(-1)^{r} \cdot s_{\left(1^{r}\right)}^{q}\left(x_{1}, \ldots, x_{k}\right) .
$$

Calculations. To illustrate the Murnaghan-Nakayama rule in the quantum cohomology of the complete flag manifold we begin with an example. Let $\omega=81253467 \in S_{8}$. Then the product of $p_{4}^{q}\left(x_{1}, \ldots, x_{4}\right)$ and $\mathfrak{S}_{w}^{q}(x)$ in the ring $q H^{*}(F l(8))$ is

$$
\begin{gathered}
q_{4} \mathfrak{S}_{\omega \cdot(3,7,6,5,4)}^{q}(x)-q_{4} \mathfrak{S}_{\omega \cdot(2,3,7,6,5)}^{q}(x)-q_{4} \mathfrak{S}_{\omega \cdot(2,3,6,5,4)}^{q}(x)-q_{4} \mathfrak{S}_{\omega \cdot(2,4,3,6,5)}^{q}(x) \\
-\mathfrak{S}_{\omega \cdot(2,5,6,4,3)}^{q}(x)+q_{1} q_{2} q_{3} q_{4} \mathfrak{S}_{\omega \cdot(1,2,4,3,8)}^{q}(x)+q_{4} \mathfrak{S}_{\omega \cdot(1,2,3,6,5)}^{q}(x)+q_{4} \mathfrak{S}_{\omega \cdot(1,2,3,5,4)}^{q}(x) \\
+q_{4} \mathfrak{S}_{\omega \cdot(1,2,4,3,5)}^{q}(x)-q_{1} q_{2} q_{3} q_{4} \mathfrak{S}_{\omega \cdot(1,2,3,5,8)}^{q}(x)
\end{gathered}
$$

Computer calculations were made for all such products in the rings $q H^{*}(F l(n))$ for $n \leq 9$ and power sums of degree $r \leq 4$. 
General Formula. Based on these calculations we suggest that the general form of the MurnaghanNakayama rule in the quantum cohomology of the complete flag manifold will be,

$$
p_{r}^{q}\left(x_{1}, \ldots, x_{k}\right) \cdot \mathfrak{S}_{\omega}^{q}(x)=\sum(-1)^{\epsilon_{k}(\sigma)} q^{w(\sigma)} \mathfrak{S}_{\omega \cdot \sigma}(x),
$$

where the sum is over cycles $\sigma$ such that $\omega$ is connected to $\omega \cdot \sigma$ via a path $\left\{\left(a_{i} b_{i}\right)\right\}$ of length $r$ in the quantum $k$-Bruhat graph. The sign $\epsilon_{k}$ is as in the classical case. The monomial factor $q^{w(\sigma)}$ is determined by a product of the contributions coming from the transpositions $\left\{\left(a_{i} b_{i}\right)\right\}$ in the path, each of which contributes a factor

$$
\begin{aligned}
1 & \text { if } l\left(\omega^{(i)}\right)=l\left(\omega^{(i-1)}\right)+1 \\
q_{a_{i}} q_{a_{i}+1} \cdots q_{b_{i}-1} & \text { if } l\left(\omega^{(i)}\right)=l\left(\omega^{(i-1)}\right)-2\left(b_{i}-a_{i}\right)+1 .
\end{aligned}
$$

We have proven this result when $r=2$. In general, Mészáros, Panova, and Postnikov have shown ([24] Theorem 8.) that in the quantum deformation of the Formin-Kirillov algebra, the quantum hook Schur polynomials can be written as a certain sum over forests. From their expressions it is immediate that the alternating sum of these polynomials simplifies to a sum over trees. Each such term corresponds to a path in the quantum Bruhat graph and also to a cycle $\sigma$ contributing with a sign $(-1)^{\epsilon_{k}(\sigma)}$. The quantum parameter $q^{w(\sigma)}$ is also immediate from the action of the Formin-Kirillov on the quantum cohomology.

For the completed version of this abstract, we hope to extend these formulas to all partial flag manifolds in type $A$, and possibly also to the $K$-theory of the flag manifold that is, to Grothendieck polynomials. Computer calculations suggest that together with the Pieri formulas of [20] one can obtain similar formulas for Grothendieck polynomials.

\section{Acknowledgements}

The author benefitted from talking with F. Sottile while a postdoc at MSRI in the Spring 2013 program. He also greatly appreciates his help in navigating the Schubert calculus literature.

\section{References}

[1] J. Bandlow, A. Schilling and M. Zabrocki, The Murnaghan-Nakayama rule for $k$-Schur functions, J. Combin. Theory Ser. A, 118 (2011), no. 5, 1588-1607.

[2] N. Bergeron and F. Sottile, A Pieri-type formula for isotropic flag manifolds, Trans. Amer. Math. Soc., 82 (2002) 2659-2705.

[3] I. N. Bernstein, I. M. Gelfand, and S. I. Gelfand, Schubert cells and cohomology of the spaces $G / P$, Russian Mathematical Surveys, 28 (1973), pp. 1-26.

[4] A. Bertram, Quantum Schubert Calculus, Adv. Math., 128 (1997), no. 2, 289-305.

[5] A. Bertram and I. Ciocan-Fontanine and William Fulton, Quantum Multiplication of Schur Polynomials, J. Algebra, 219 (1999), no. 2, 728-746.

[6] I. Ciocan-Fontanine, On quantum cohomology rings of partial flag varieties, Duke Math. J., 98 (1999), no.3, 485-524. 
[7] I. Ciocan-Fontanine, Quantum cohomology of flag varieties, Internat. Math. Res. Notices, 6 (1995) 263-277.

[8] M. Demazure, Désingularization des variétés de Schubert généralisées, Ann. Sc. E. N. S. (4), 7 (1974), pp. 53-88.

[9] W. Fulton and C. Woodward, On the quantum product of Schubert classes, J. Algebraic Geom., 13 (2004), 641-661.

[10] W. Fulton and J. Harris, Representation Theory: A first course, Graduate Texts in Mathematics, Vol. 129, Springer, 2004.

[11] W. Fulton, Young Tableaux: with applications to representation theory and geometry, LMS Student Texts, 35, Cambridge University Press, 1997

[12] S. Fomin and C. Greene, Noncommutative Schur functions and their applications, Discrete Math., 193 (1998), no. 1-3, 179-200.

[13] S. Fomin, S. Gefland and A. Postnikov, Quantum Schubert polynomials, J. Amer. Math. Soc., 10 (1997), 565-596.

[14] W. Fulton and R. Pandharipande, Notes on stable maps and quantum cohomology, Proc. Sympos. Pure Math., 1997

[15] M. Goodman and H. Wenzl, Littlewood-Richardson coefficients for Hecke algebras at roots of unity , Adv. Math., 82 (1990), no.2, 244-265.

[16] M. Konvalinka, Skew quantum Murnaghan-Nakayama rule, J. Algebraic Combin., 35 (2012), no.4, 519-545.

[17] V. Kac, Infinite-Dimensional Lie Algebras, 3rd Ed., Cambridge University Press, 1990.

[18] B. Kim, Quantum cohomology of flag manifolds. G/B and quantum Toda lattices, Ann. of Math., 149, (1999), 129-148.

[19] A. Lascoux and M-P. Schützenberger, Polynômes de Schubert, C. R. Acad. Sci. Paris Sér. I Math., 294 (1982), no. 13, 447-450.

[20] L. Cristian and F. Sottile, A Pieri-type formula for the $K$-theory of a flag manifold., Trans. Amer. Math. Soc. 359, no. 5 (2007), 2317-2342.

[21] I.G. Macdonald, Symmetric functions and Hall polynomials, Oxford Mathematical Monographs, 1995.

[22] I.G. Macdonald, Notes on Schubert Polynomials, Laboratoire de combinatoire et d'informatique mathématique (LACIM), Univ. du Québec à Montréal, Montréal, 1991.

[23] P. McNamara, A Pieri Rule for Skew Shapes, Talk at Formal Power Series and Algebraic Combinatorics, San Francisco, 4th August 2010. 
[24] K. Meszaros, G. Panova, A. Postnikov, Schur times Schubert via the Fomin-Kirillov algebra, arXiv:1210.1295, accepted Electronic J. Combinatorics, (2013).

[25] D. Monk, The Geometry of Flag Manifolds, Proc. London Math. Soc. (3) 9 (1959), 253-286.

[26] A. Postnikov, On a quantum version of Pieri's formula, Advances in geometry, Progr. Math., vol. 172, Birkhäuser Boston, Boston, MA, 1999, pp. 371-383.

[27] D. Ross, The loop Murnaghan-Nakayama rule, accepted Journal of Algebraic Combinatorics, (2013).

[28] D. Ross and Z. Zong, The Gerby Gopakumar-Marino-Vafa Formula, Geometry and Topology, 17 (2013), no. 5. 2935-2976.

[29] F. Sottile, Pieri's formula for flag manifolds and Schubert polynomials , Ann. Inst. Fourier (Grenoble), 46, (1996) no. 1, 89-110.

[30] M. Walton, Fusion rules in Weiss-Zumino-Witten models, Nuclear Phys. B, 340 (1990), no. 2-3, 777-790.

[31] E. Witten, The Verlinde Algebra And The Cohomology Of The Grassmannian, Conference Proceedings and Lecture Notes in Geometric Topology, (1995). 